\title{
Enhancement of Gas Sensing Properties through Branch Formation and Metal Catalysts
}

\author{
Myung Sik Choi', Jae Hoon Bang ${ }^{1}$, Wansik Oum ${ }^{1}$, Ali Mirzaei², Sang Sub Kim ${ }^{3, *}, \underline{\text { Hyoun Woo Kim }}^{1,2, *}$ \\ ${ }_{1}^{1}$ Division of Materials Science and Engineering, Hanyang University, Seoul, 133-791, \\ Republic of Korea \\ ${ }^{2}$ The Research Institute of Industrial Science, Hanyang University, Seoul 04763, \\ Republic of Korea \\ ${ }^{3}$ Department of Materials Science and Engineering, Inha University, Incheon 22212, \\ Republic of Korea \\ hyounwoo@hanyang.ac.kr, sangsub@inha.ac.kr
}

\begin{abstract}
Nanowires have been studied and used as sensing materials because of their outstanding sensitivity and selectivity. Also decorating nanomaterials with branches and metal nanoparticles has been done for using their heterointerfaces' reactions and catalytic effects. In this experiment, we report a novel method to improve the gas sensing properties of metal oxide nanowires through the interactions of metal oxide branches and metal nanoparticles.
\end{abstract}

Key words: Nanowires, branches, metal nanoparticles, gas sensing

\section{Background}

Recently, there are several environmental threats and especially problems with toxic gases are important social issues. To solve the problem, researchers have experimented gas sensors using many kinds of semiconductors. Also to detect the low concentration of toxic gases, lots of experiments have been concentrating their focus on manipulating morphologies of sensing materials [1,2]. In here, we fabricated $\mathrm{SnO}_{2}$ stem nanowires and metaloxide branches were grown on the surface of $\mathrm{SnO}_{2}$ nanowires. Furthermore, metal nanoparticles were attached on the surface of as fabricated nanowires. For sensing tests, interdigitated $\mathrm{Au}$ top electrode was deposited on the specimens. Gas sensing properties of as-fabricated sensor were enhanced by their resistance modulation by the branch formation and catalytic effect by metal nanoparticles.

\section{Experimental}

The fabrication of $\mathrm{Au}$ functionalized $\mathrm{TeO}_{2}-$ branched $\mathrm{SnO}_{2}$ nanowire is as follows. First, we fabricated $\mathrm{SnO}_{2}$ nanowires by thermal evaporation of Sn powder. Sn powder (purity: $99.9 \%$, Sigma-Aldrich) was used as the source material. The substrate temperature was set to $900^{\circ} \mathrm{C}$ for $1 \mathrm{hr}$ to heat $3 \mathrm{~nm}-\mathrm{Au}$ coated Si substrates. A mixture of $\mathrm{Ar}$ and $\mathrm{O}_{2}$ gases $\left(\mathrm{O}_{2}\right.$ : $3 \%$; Ar: $97 \%$ ) was set at a fixed 2 Torr pressure. To fabricate $\mathrm{TeO}_{2}$-branched $\mathrm{SnO}_{2}$ nanowires, 3nm-Au was coated again onto asfabricated $\mathrm{SnO}_{2}$ nanowires. Then we fabricated $\mathrm{TeO}_{2}$-branched $\mathrm{SnO}_{2}$ nanowires by thermal evaporation of Te powder (purity: 99.99\%, Sigma-Aldrich). At $370^{\circ} \mathrm{C}$, Te powders were evaporated and combined with oxygen. Finally to fabricate Au nanoparticles, Au thin film (3nm) were sputtered on the surface of as fabricated $\mathrm{TeO}_{2}$-branched $\mathrm{SnO}_{2}$ nanowire and annealed at $300^{\circ} \mathrm{C}$ with $\mathrm{Ar}$ gases.

\section{Results}

Figure 1 shows SEM images of $\mathrm{SnO}_{2}$ nanowire, $\mathrm{TeO}_{2}$-branched $\mathrm{SnO}_{2}$ nanowire, and $\mathrm{Au}$ nanoparticle functionalized $\mathrm{TeO}_{2}$-branched $\mathrm{SnO}_{2}$ nanowire. $\mathrm{TeO}_{2}$ branches and $\mathrm{Au}$ nanoparticles were grown randomly on the surface of $\mathrm{SnO}_{2}$ nanowires. Figure 2 shows gas response of $\mathrm{SnO}_{2}$ nanowire, $\mathrm{TeO}_{2}$ branched $\mathrm{SnO}_{2}$ nanowire, and $\mathrm{Au}$ functionalized $\mathrm{TeO}_{2}$ branched $\mathrm{SnO}_{2}$ nanowire to $\mathrm{NO}_{2}$ gas. Gas response of branched $\mathrm{SnO}_{2}$ nanowires is higher than that of bare $\mathrm{SnO}_{2}$ nanowires. Also with metal functionalization, gas response was increased. 

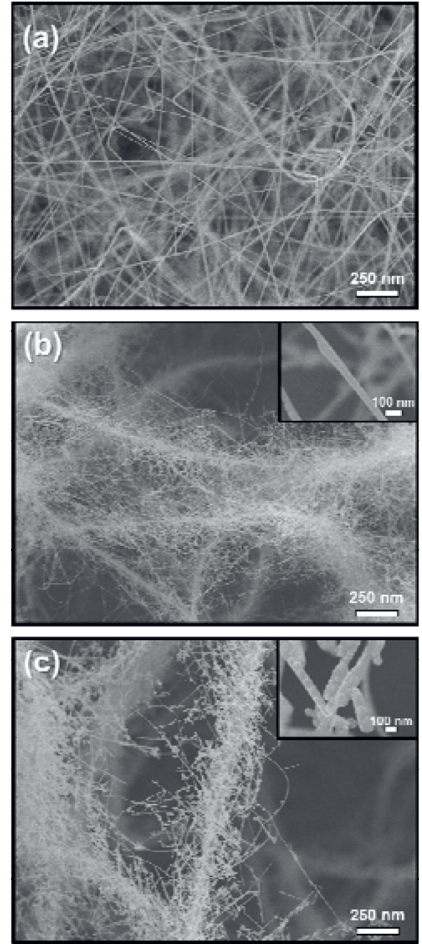

Fig. 1. SEM images of (a) $\mathrm{SnO}_{2}$ nanowire, (b) $\mathrm{TeO}_{2}$-branched $\mathrm{SnO}_{2}$ nanowire, and (c) $\mathrm{Au}$ functionalized $\mathrm{TeO}_{2}$-branched $\mathrm{SnO}_{2}$ nanowire.
Fig. 2. Gas response of (a) $\mathrm{SnO}_{2}$ nanowire, (b) $\mathrm{TeO}_{2}$-branched $\mathrm{SnO}_{2}$ nanowire, and (c) $\mathrm{Au}$ functionalized $\mathrm{TeO}_{2}$-branched $\mathrm{SnO}_{2}$ nanowire to $\mathrm{NO}_{2}$ gas.

\section{References}

[1] A. Sarkar, K. Kanakamedala, N. N. Jagadish, A. Jordan, S. Das, N. Siraj, I. M. Warner, T. DanielsRace, Electro-optical characterization of cyaninebased GUMBOS and nanoGUMBOS. Electron. Mater. Lett. 10, 879-885 (2014);

[2] Q. Wan, J. Huang, Z. Xie, T. H. Wang, E. N. Dattoli, W. Lu, Branched $\mathrm{SnO}_{2}$ nanowires on metallic nanowire backbones for ethanol sensors application. Appl. Phys. Lett. 92, 102101 (2008);
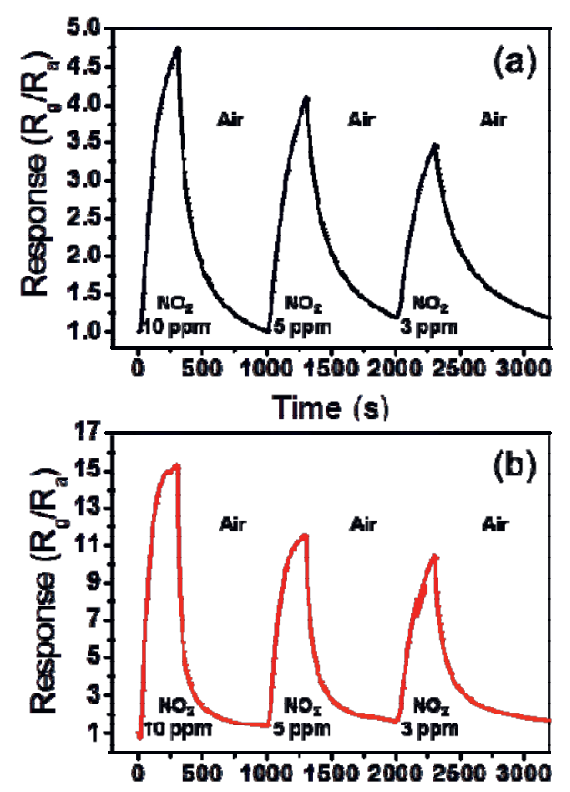

Time (s)

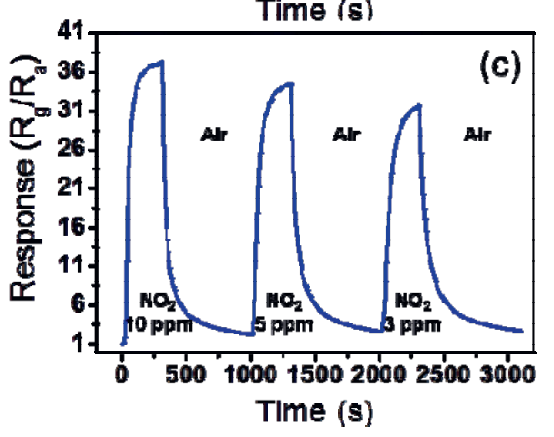

